INPLASY

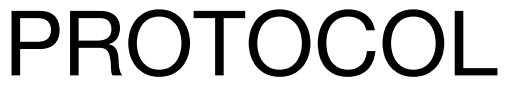

To cite: Du et al.

Postdiagnostic aspirin use and prognosis of breast cancer: a meta-analysis of cohort studies. Inplasy protocol 202220075. doi:

10.37766/inplasy2022.2.0075

Received: 18 February 2022

Published: 19 February 2022

Corresponding author:

Qiaoling Song

qiaoling.song@zcmu.edu.cn

Author Affiliation:

The First School of Clinical

Medicine of Zhejiang Chinese

Medical University.

Support: None.

Review Stage at time of this submission: Data analysis Completed but not published.

Conflicts of interest:

None declared.

\section{Postdiagnostic aspirin use and prognosis of breast cancer: a meta-analysis of cohort studies}

Du, W1; Guo, K2; Jin, H³; Sun, L4; Ruan, S5; Song, Q6.

Review question / Objective: Previous studies suggested that aspirin may prevent the incidence of breast cancer. However, it remains poorly understood that if concurrent use of aspirin in women with breast cancer is associated with improved clinical outcomes. The aim of the study is to perform a metaanalysis to assess the influence of postdiagnostic aspirin use on the recurrence and disease-specific mortality in women with breast cancer.

Condition being studied: Although some cohort studies showed that postdiagnostic aspirin use might be related to reduced recurrence or disease-specific survival in women with breast cancer, others did not. Besides, two previous meta-analyses assessing the influence of NSAIDs/aspirin on clinical outcomes of women with breast cancer were performed more than five years ago, and multiple related observational studies have been published since then. Therefore, we aimed to perform an updated meta-analysis of cohort studies to systematically observe the possible impact of postdiagnostic aspirin use on the clinical outcomes in women with breast cancer.

INPLASY registration number: This protocol was registered with the International Platform of Registered Systematic Review and Meta-Analysis Protocols (INPLASY) on 19 February 2022 and was last updated on 19 February 2022 (registration number INPLASY202220075).

\section{INTRODUCTION}

Review question / Objective: Previous studies suggested that aspirin may prevent the incidence of breast cancer. However, it remains poorly understood that if concurrent use of aspirin in women with breast cancer is associated with improved clinical outcomes. The aim of the study is to perform a meta-analysis to assess the influence of postdiagnostic aspirin use on the recurrence and disease-specific mortality in women with breast cancer.

Condition being studied: Although some cohort studies showed that postdiagnostic aspirin use might be related to reduced recurrence or disease-specific survival in 
women with breast cancer, others did not. Besides, two previous meta-analyses assessing the influence of NSAIDs/aspirin on clinical outcomes of women with breast cancer were performed more than five years ago, and multiple related observational studies have been published since then. Therefore, we aimed to perform an updated meta-analysis of cohort studies to systematically observe the possible impact of postdiagnostic aspirin use on the clinical outcomes in women with breast cancer.

\section{METHODS}

Search strategy: Electronic literature databases including PubMed, Web of Science, and Embase will be systematically searched with a strategy based on combined key words such as: (1) "breast cancer" (2) "aspirin" OR "antiplatelet" OR "NSAIDs" OR "nonsteroidal antiinflammatory drugs"; and (3) "recurrence" OR "survival" OR "mortality" OR "death" OR "progression" OR "relapse" OR "metastasis" OR "prognosis".

Participant or population: Women with breast cancer.

Intervention: Women with postdiagnostic aspirin use.

Comparator: Women without postdiagnostic aspirin use.

Study designs to be included: Cohort studies.

Eligibility criteria: Studieswill be included if they met the following criteria: (1) published as full-length article; (2) designed as cohort studies with the minimal followup duration of one year; (3) included women with breast cancer; (4) postdiagnostic use of aspirin was identified as exposure of interest; (5) documented the incidence of breast cancer recurrence or breast cancer mortality during follow-up; and (6) reported the adjusted risk ratios (RRs, at least adjusted for age) and their corresponding $95 \%$ confidence intervals (CIs) for the above outcomes comparing breast cancer women with and without the use of aspirin.

Information sources: Electronic literature databases including PubMed, Web of Science, and Embase will be systematically searched with a strategy based on combined key words such as: (1) "breast cancer" (2) "aspirin" OR "antiplatelet" OR "NSAIDs" OR "nonsteroidal antiinflammatory drugs"; and (3) "recurrence" OR "survival" OR "mortality" OR "death" OR "progression" OR "relapse" OR "metastasis" OR "prognosis". Only studies with human subjects and published in English language were included. We will also screen the citation lists of the related original and review papers in a manual manner as a complementation.

Main outcome(s): Breast cancer recurrence or incidence of breast cancer mortality with the follow-up for at least one year; reported as the adjusted risk ratios (RRs) and 95\% confidence intervals (Cls) at least for age.

Quality assessment / Risk of bias analysis: The quality of each study will be evaluated using the Newcastle-Ottawa Scale (NOS). This scale ranges from 1 to 9 stars and judges the quality of each study regarding three aspects: selection of the study groups; the comparability of the groups; and the ascertainment of the outcome of interest.

Strategy of data synthesis: The associations between postdiagnostic aspirin use and breast cancer recurrence and mortality will be measured by RRs in this study. To stabilize its variance and normalized the distribution, RR data and its corresponding stand error (SE) from each study will be logarithmically transformed. The Cochrane's $\mathbf{Q}$ test will be performed to evaluate the heterogeneity among the include cohort studies, and the 12 statistic will be also calculated. A significant heterogeneity will be considered if $12>$ $50 \%$. A random effect model will be used to pool the results since this model has been indicated to incorporate the potential heterogeneity of the included studies and therefore could provide a more generalized 
result. Predefined subgroup analysis will be used to evaluate potential study characteristics on the outcome, including study design and adjustment of hormonal receptor status of the tumor. Potential publication bias will be assessed by visual inspection of the symmetry of the funnel plots, complemented with the Egger regression test. The RevMan (Version 5.1; Cochrane Collaboration, Oxford, UK) and STATA software will be used for the statistics.

Subgroup analysis: Predefined subgroup analysis will be used to evaluate potential study characteristics on the outcome, including study design and adjustment of hormonal receptor status of the tumor.

Sensitivity analysis: Sensitivity analysis by omitting one study at a time will be performed to evaluate the robustness of the findings.

\section{Language: English.}

Country(ies) involved: China.

Keywords: Breast cancer; Aspirin; Recurrence; Mortality; Meta-analysis.

Contributions of each author:

Author 1 - Wurong Du - conceived and designed research; collected data and conducted research; performed or supervised analyses; interpreted data; wrote the initial paper. provided substantive suggestions for revision, reviewed and approved final version of the paper.

Email: duwurong214@163.com

Author 2 - Kaibo Guo - conceived and designed research; collected data and conducted research; interpreted data; provided substantive suggestions for revision, reviewed and approved final version.

Email: guokaibo@zcmu.edu.cn

Author 3 - Huimin Jin - conceived and designed research; provided substantive suggestions for revision, reviewed and approved final version.

Email: huiminjin@zcmu.edu.cn

Author 4 - Leitao Sun - interpreted data; provided substantive suggestions for revision, reviewed and approved final version.

Author 5 - Shanming Ruan - interpreted data; provided substantive suggestions for revision, reviewed and approved final version.

Email: shanmingruan@zcmu.edu.cn

Author 6 - Qiaoling Song - conceived and designed research; performed or supervised analyses; interpreted data; provided substantive suggestions for revision, reviewed and approved final version.

Email: qiaoling.song@zcmu.edu.cn 